\title{
Performance Studies of MPLS Based Integrated Architecture for 3G-WLAN Scenarios with QoS Provisioning
}

\author{
${ }^{1}$ Iti Saha Misra and ${ }^{2}$ Chandi Pani \\ ${ }^{1}$ Dept. of Electronics and Telecommunication Engg., Jadavpur University, Kolkata, India \\ ${ }^{2}$ Dept. of Electronics and Communication Engineering \\ Meghnad Saha Institute of Technology, Kolkata-700150, India \\ litimisra@cal.vsnl.net.in, ${ }^{2}$ chandi_pani2309@yahoo.co.in
}

\begin{abstract}
Recently, the growing need of telecommunications such as VideoConference, Voice over IP etc. and for the diversity of transported flows, Internet network does not meet the requirements of future integrated services satisfying Quality-of-Services (QoS). This is become much more complex for heterogeneous networks especially for wireless networks. In this paper, we propose an MPLS based integrated architecture between 3G(UMTS)/WLAN networks. We present the performance analysis of this MPLS enabled 3GWLAN integrated framework for better QoS, throughput, less switching delay and less packet loss with the aim to fulfill the future demand of wireless communication. The mobility of the integrated framework is managed using hierarchical MIPv6 (HMIPv6). Integration point is considered at the MAP (Mobility Anchor Point) to restrict global update. Extensive simulation is done on mns-2.0 to evaluate the network performance in terms of packet forwarding, throughput and delay. For traffic engineering in the MPLS domain, CRLDP is used.
\end{abstract}

Keywords: 3G/WLAN integration, MPLS, UMTS, Traffic Engineering and QoS.

\section{Introduction}

In this paper end-to-end communications over an information transport consisting of heterogeneous communication platform mainly 3G/UMTS cellular networks and WLAN is provided. Mobile operators are transitioning towards third generation (3G) or beyond in order to access high-speed data rate in conjunction with IP. WLAN [1] provides high data rate with least installation cost but one of the major drawback of WLAN is limited area of coverage. Cellular systems [2] are one of the popular wireless access technologies in communication domain. It has moved through different generations like 1G, 2G (GSM, DAMPS), 2.5G (GPRS, EDGE) followed by 3G (UMTS) [2]. In 1992, International Telecommunication Union (ITU) has issued International Mobile Telecommunication for year 2000 (IMT-2000) which defines the basic characteristics of $3 \mathrm{G}$. But due to the high deployment cost, $3 \mathrm{G}$ has not been accepted globally into the market. Advantages of cellular system are wide coverage

Please use the following format when citing this chapter:

Misra, I. S., Pani, C., 2007, in IFIP International Federation for Information Processing, Volume 245, Personal Wireless Communications, eds. Simak, B., Bestak, R., Kozowska, E., (Boston: Springer), pp. 157-168. 
and well known voice service, whereas limitations are low data rate and more costly compared with WLAN. The complementary characteristics of $3 \mathrm{G}$ cellular systems (slow, wide coverage) and WLAN (fast, limited coverage) make it attractive to integrate these two technologies to provide ubiquitous wireless access.

\subsection{Focus of Integration}

Considering the advantages and disadvantages of WLAN and Cellular systems, aim of integration is to develop a network, comprising the best features of these two heterogeneous technologies. The integration model will allow 3G/UMTS users to access the WLAN services from hotspot regions whenever they require high data rate and switch back in 3G/UMTS region when the service quality of the WLAN is not satisfactory or they are outside the WLAN region.

\subsection{Related Work}

There are numerous proposals regarding integration of these two technologies. [3] is the proposition of integration based on tight coupling (TCIA). But it has several disadvantages: TCIA inject the WLAN traffic directly towards the core network increasing the overall traffic in the core network. Secondly, the core network is completely exposed to WLAN network which violets the security and privacy of cellular network. Integration based on loose coupling (LCIA) [4] is introduced to overcome the disadvantage of TCIA. LCIA use mobile IP (MIP) [5] to provide seamless mobility. MIP has some disadvantage of triangular routing and seamless mobility in hand off intensive environment. So, MIP based integration introduces significant network overhead in terms of increased delay, packet loss and signaling when the user changes its point of attachment very quickly. To overcome these deficiencies hierarchical mobile IP (HMIP) based protocols [6-8] have been proposed which divides the network into domain and domains are further subdivided into subnets. HMIPv6 [9] is based on MIPv6 platform that introduces a new entity called mobility anchor point (MAP), the major idea is that once mobile node (MN) registers with MAP's CoA with home agent (HA), there is no requirement of further registration when MN moves locally i.e within the MAP. So this method provides low signaling overhead and less number of location update.

Multi protocol label switching (MPLS) [10] is a label-forwarding scheme provides a better solution for faster switching. MPLS is a versatile solution to address the problems faced by present-day networks-speed, scalability, quality-of-service (QoS) management, and traffic engineering. MPLS has emerged as an elegant solution to meet the bandwidth-management and service requirements for next-generation IPbased backbone networks. When it is used in conjunction with IP, conventional IP look up and forwarding within the network is replaced by faster label look up and switching, the IP header is analyzed only in entry and exit points of the network. There are so many advantages of MPLS systems [11], which motivate us to use MPLS in mobile wireless communication networks. Related works that support mobility in MPLS domain is mainly based on MIPv4 and HMIPv4 [12-14]. A quality 
of service (QoS) provisioning scheme is given in [15]. Integration of MPLS with hierarchical MIP based architecture and mobility management schemes are given in $[16,17]$. It is observed that the signaling cost for traffic transport is much reduced for MPLS and hierarchical mobile IP based integrated structure [16].

The current Internet Gateway Protocol (IGP) use shortest paths to forward traffic. It causes some of the links to be over utilized and some are under utilized. The purpose of traffic engineering (TE) $[18,19]$ is to enhance network utilization and to improve architecture of a network in a systematic way, so that the network becomes robust, adaptive and easy to operate. MPLS has extended routing capability that efficiently controls the network traffic by removing congestion and spreading the load over the different links. In [20-22] different route selection algorithms based on MPLS framework is provided. LDP (Label Distribution Protocol) is a new protocol that defines a set of procedures and messages by which one LSR (Label Switched Router) informs another of the label bindings it has made. CR-LDP [21] (constraintbased LDP) contains extensions for LDP to extend its capabilities. This allows extending the information used to setup paths beyond what is available for the routing protocol.

\subsection{Motivation}

The development and standardization process are currently underway for defining suitable efficient integrated architectures and are a challenging task hat needs a lot of research efforts. The growing worldwide deployment of public WLANs has a growing impact on what public wireless networks will look like and how public mobile services will be provided in the near future. WLAN provide significantly higher data rates than cellular networks that are expected to be available in the near future [23]. Public WLANs are the first wave of all-IP radio access networks making one step forward on their migrations to IP based wireless networks. It calls for new and innovative business models for public mobile services. $3 \mathrm{G}$ wireless networks allow mobile users to access Internet via standard IP with higher data rates. As the demand for multimedia data services increases, end users aim for enhanced performance through the greater coverage, higher data rate and lower overall cost. Whereas, mobile operators aims in generating large cost effective business to a wide range of applications such as mobile multimedia services (MMS). To meet this demand it requires the suitable integration of the existing networks maintaining the end-to-end QoS for data services. Moreover, the integration of QoS parameters will increase complexity in heterogeneous networks that include different types of networks (wired, wireless, mobile etc.).

Thus, in this paper we are exploring an integrated architecture between the $3 \mathrm{G}$ WLAN paradigms for enhanced performance. We choose MPLS as the best choice for high switching rate and QoS maintenance. The proposed framework uses the enhanced type of MPLS router called label edge mobility agent (LEMA) that are placed at different hierarchical level of the integrated framework. LEMA takes an active part in mobility management. In the proposed model, MAP is placed at the integration point of the network that restricts the movement of the mobile node under MAP domain between the two heterogeneous networks. Use of MPLS in the proposed 
framework can satisfy the seamless mobility with fast packet forwarding, fast hand off, less number of packet loss during hand off and minimum delay that make the framework more scalable.

\section{Proposed MPLS/HMIPv6 based Integrated Model and Mobility Management}

Fig.1 shows the MPLS/HMIPv6 based integrated framework where two heterogeneous networks UMTS and WLAN are considered as two different domains. In the proposed architecture, MAP is placed at the integration point of two heterogeneous network to reduce signaling overhead and location update.

From the aspect of scalability, fast packet forwarding and QoS based Traffic engineering, MPLS domain is formed between MAP and the two heterogeneous networks where MAP is the ingress and 3G-AR, WGWR are the egress LER (Label edge router). So, all the edge routers have LEMA property and they are named as MAP LEMA, 3G-AR LEMA and WGWR LEMA whereas the intermediate routers are the LSR. Mobility of the network is handled by HMIPv6 protocol. Mobile IP initiates the MIP registration message to establish LSP. For signaling protocol, CRLDP is used.

During registration, all the LEMAs are modified their label forwarding information base (LFIB) by placing the care of address (CoA) of MN in the forward equivalent class (FEC), also modify their label forwarding table (LFT) by placing the in-label and out-label value for next hop information and a new label switch path (LSP) is established afterward [17]. For seamless mobility to occur the user equipment (UE) must have dual mode terminal to interface 3G/UMTS and 802.11b.The 802.11b interface allow the user to operate in WLAN domain and UMTS driver allow the user to operate in UMTS domain. The different protocol stacks of integrated framework are shown in Fig.2. To describe mobility management, firstly it considers mobile node (MN) is under AR1 of UMTS domain. For intra domain mobility the movement of MN is restricted under same domain, 3G-AR LEMA handles the mobility issues, no requirement to inform MAP LEMA or HA. When MN moves from UMTS to WLAN domain, the mobility is inter domain mobility and managed by MAP LEMA. When MN moves outside the range of MAPLEMA, new registration up to HA be required henceforth, it is called the global mobility. After the registration process for the $\mathrm{MN}$, CR-LDP is employed for establishment of traffic path. For dynamic route, CRLSP (Constrain Routing Label Switched Path) is configured to provide QoS and to follow automatic reconfiguration when a failure occur or the network state changes. 


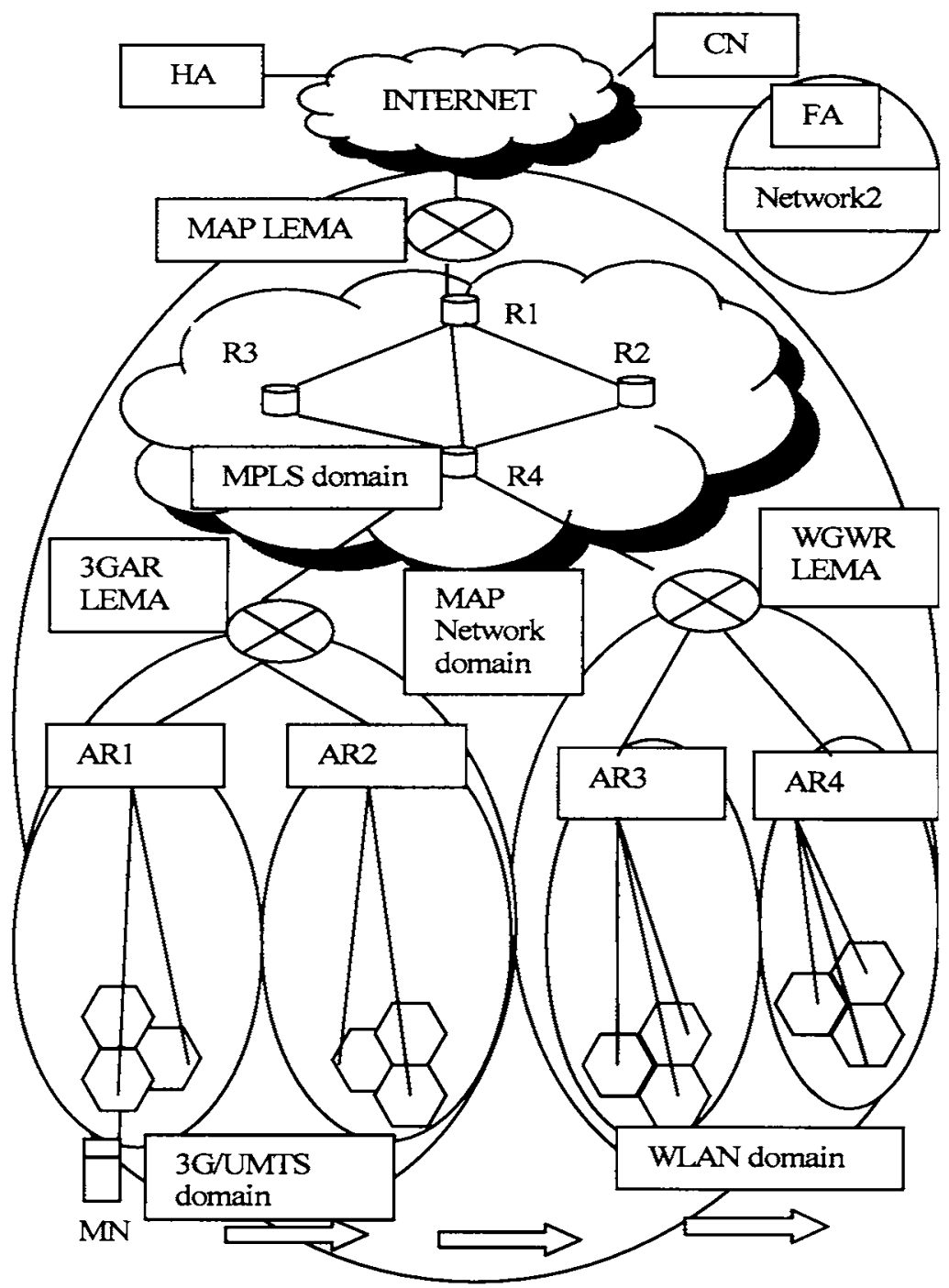

HA-home agent, CN- correspondent node, MAPLEMA-MPLS enabled MAP, 3GARLEMA-MPLS enabled 3G/UMTS gateway LEMA, WGWRLEMA-MPLS enabled WLAN gateway LEMA, MN-mobile node, AR- Access router, BSC- base station controller

O MPLS Router $\bigcirc$ Access point.

Fig. 1 Proposed integrated framework 


\begin{tabular}{|c|c|}
\hline \multicolumn{2}{|c|}{ 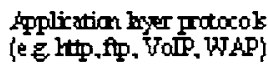 } \\
\hline \multicolumn{2}{|c|}{$\begin{array}{l}\text { Trempoort lyee protocais } \\
\text { (AZ TCP,UDP\} }\end{array}$} \\
\hline \multicolumn{2}{|c|}{ 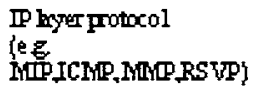 } \\
\hline $802.1 \mathrm{~J} \mathrm{LLC}$ & \multirow{3}{*}{$\begin{array}{l}\text { Unirs } \\
\text { Devire } \\
\text { Driver }\end{array}$} \\
\hline $802.1 \mathrm{~b} \mathrm{MCAC}$ & \\
\hline $802.1 \mathrm{mb} \mathrm{PHY}$ & \\
\hline
\end{tabular}

UE

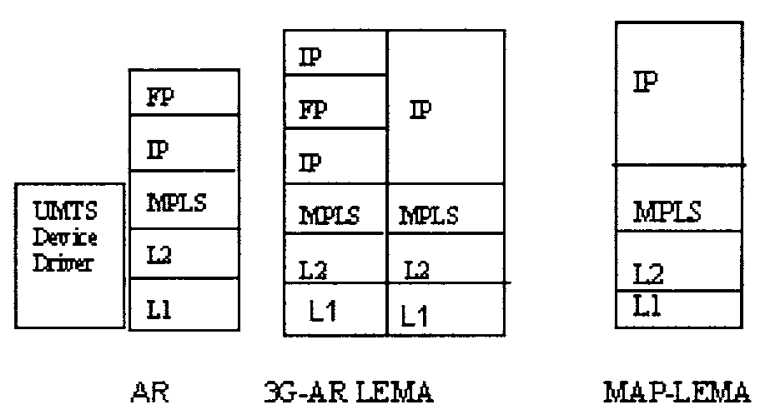

Figure 2. Protocol Stack for the integrated architecture

\section{Network Performance Evaluation Through Simulation}

We have performed series of simulations in order to evaluate the UMTS/WLAN/MPLS based architecture. The simulations are implemented using Network Simulator 2.26 (ns-2.26) [24].

The network topology as shown in Fig. 3 is used for simulation purpose. The entry node of the access network is referred to as MAP LEMA for integrated MPLS/HMIPv6 protocol. It will simply a MAP node when simulate only for HMIPv6 based mobility architecture. Within the MPLS domain, the internal nodes are LSR (Label Switched Router) otherwise they are IPv6 routers. The exit points of the domain are referred to as 3G LEMA or WGWR LEMA (for WLAN access router) for MPLS enabled architecture. For simulation, we focus on the traffic engineering and mobility management of the proposed architecture. In simulation scenario, we consider all the links have the capacity of $1 \mathrm{Mb}$ with $100 \mathrm{~ms}$ delay. We assume that packets are arrived from $\mathrm{CN}$ to $\mathrm{MN}$ with exponentially varying packet size.

\subsection{Simulation Results}

This section discusses about the simulation results for both the architecture with and without the MPLS. To examine the TE performance, we use distance vector algorithm for route selection and Figure 4. LSP set up time for two cases with three exponential traffic of packet size 200 , burst $2 \mathrm{sec}$, idle $1 \mathrm{sec}$ and rate $100 \mathrm{k}, 200 \mathrm{k}, 300 \mathrm{k}$ respectively. Three different simulation scenarios are considered as given under.

\section{Scenario 1}


Case A: $\mathrm{MN}$ is registered with ARl of Fig.3 under $3 \mathrm{G}$ domains. For traffic engineering, CR-LDP is used.

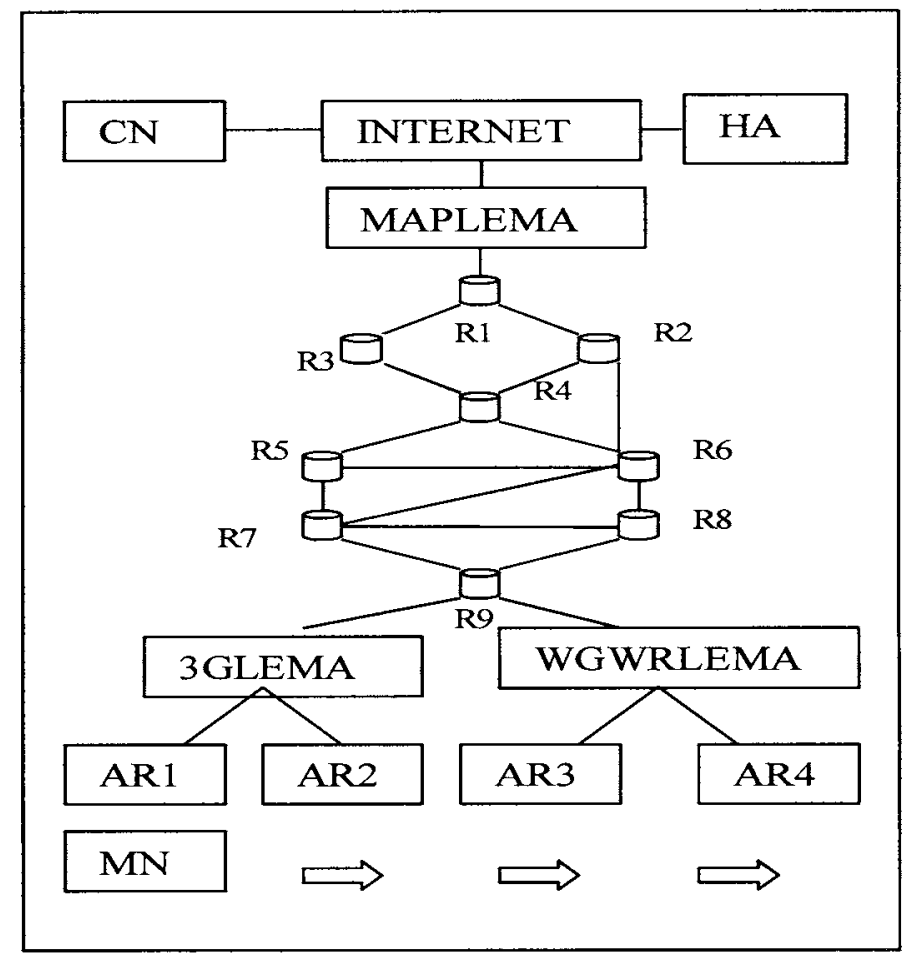

Figure 3. Simulated network

Figure 3. Network for simulation protocol is used to establish LSP in integrated MPLS domain and distant vector routing is used for HMIPv6 domain without MPLS. Fig.4 shows the path set up time from $\mathrm{CN}$ (corresponding node) to $\mathrm{MN}$ for two protocols (with MPLS, without MPLS). It is seen from Fig.4 that number of packet required to set up LSP is less in case of MPLS enabled framework. Also it requires less time for path setup.

Case B: Now three different packets having same size as mentioned earlier are sent from $\mathrm{CN}$ to $\mathrm{MN}$ under same scenario as in case A. Fig. 5 and Fig. 6 show the bandwidth consumed by MN for different protocols with and without MPLS. It is seen that in Fig. 5 only packet 3 is reached to $\mathrm{MN}$ over the simulation period whereas the other two packets are dropped. But in Figure 6, for HMIPv6 integrated network case of MPLS enabled framework all three packets are reached to MN over simulation period. Also the bandwidth consumption for packets forwarding is less in case of MPLS network. 


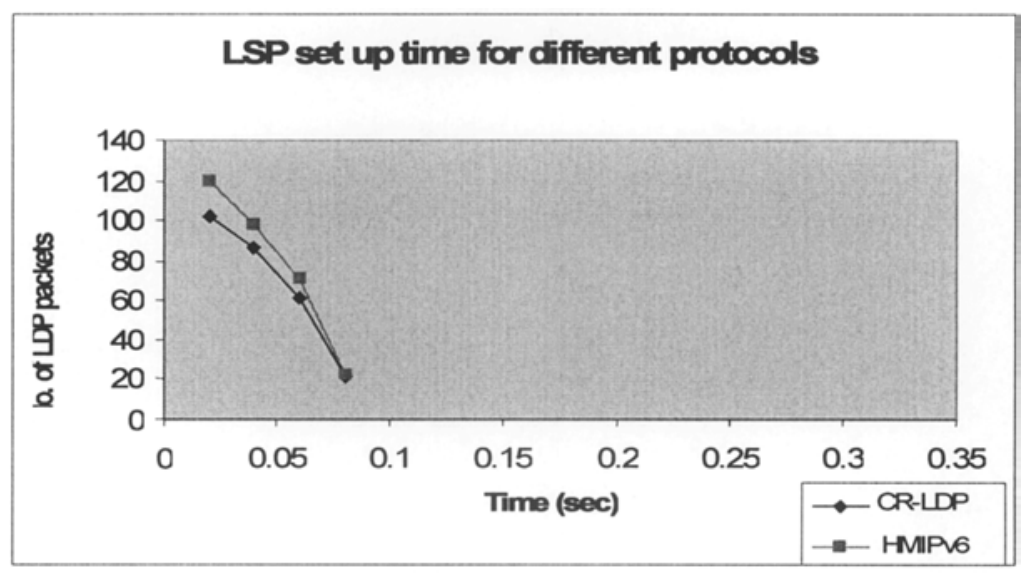

Figure 4. LSP setup time for two cases

\section{Time vs. Bandwidth consumed by HMPv6 enabled netuork}

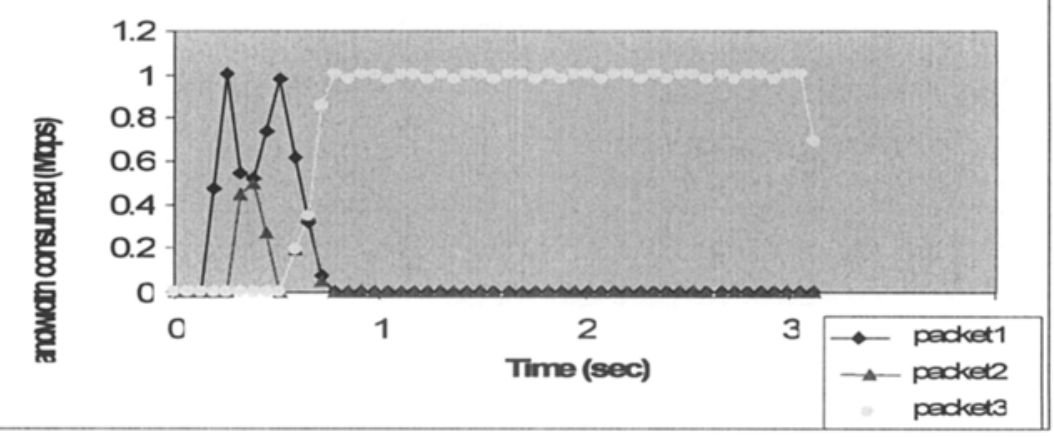

Figure. 5 Packet flow scenario for the simulation network for HMIPv6 integrated network

\section{Scenario 2}

Case A: $\mathrm{MN}$ is moved from $3 \mathrm{G}$ domains to WLAN domain (from AR2 to AR3) and same amount of packets are sent from $\mathrm{CN}$ to $\mathrm{MN}$ as considered in earlier cases. Fig.7 shows the amount of packet loss during hand off between these two domains for two different protocols and approximately $68 \%$ gain is achieved in connection with dropped packet for MPLS network.

Case B: Then we increase the packet size in Mbytes and forward it from $\mathrm{CN}$ to $\mathrm{MN}$ for the flow of $3 \mathrm{G}$ domains to WLAN domain. Fig. 8 shows the average throughput in Mbps for two cases with achieved gain is approximately $28 \%$ for MPLS enabled integrated architecture.

From all the above results it can be said that for future generation network with increased network traffic load for mobile wireless communications, MPLS enabled mobility management network may fulfill the QoS requirement for bandwidth, throughput, delay and faster switching. Our proposed network model is one way of 
integrating heterogeneous networks. As HMIPv6 is the hierarchical mobility management protocol established to reduce signaling and delay overhead, the MPLS enabled model shows the improvement of the performance with respect to HMIPv6. This is because of the inherent advantages obtained from MPLS.

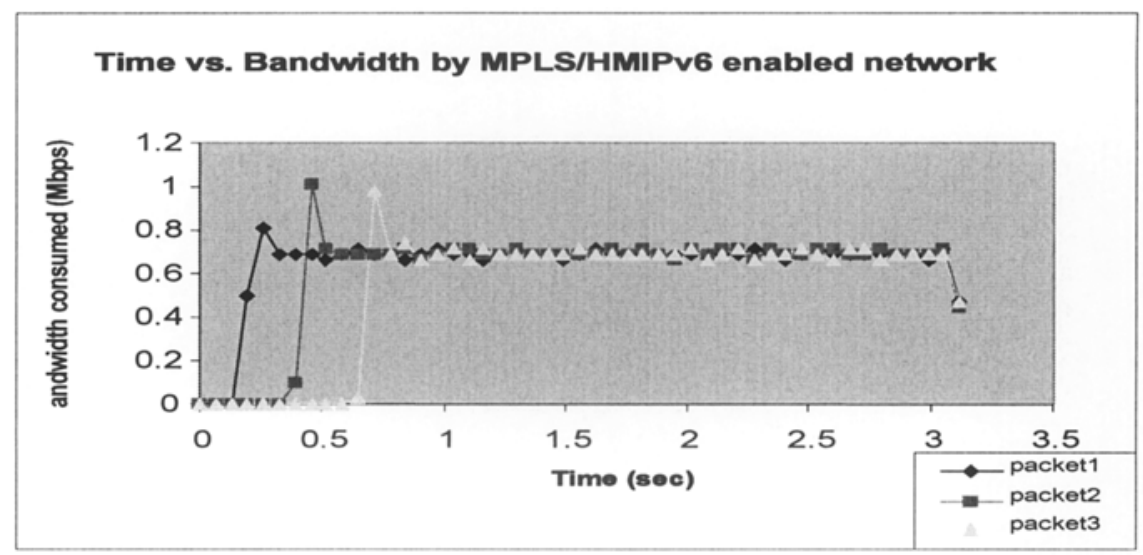

Figure 6. Packet flow scenario for the simulation network for MPLS/HMIPv6 integrated network

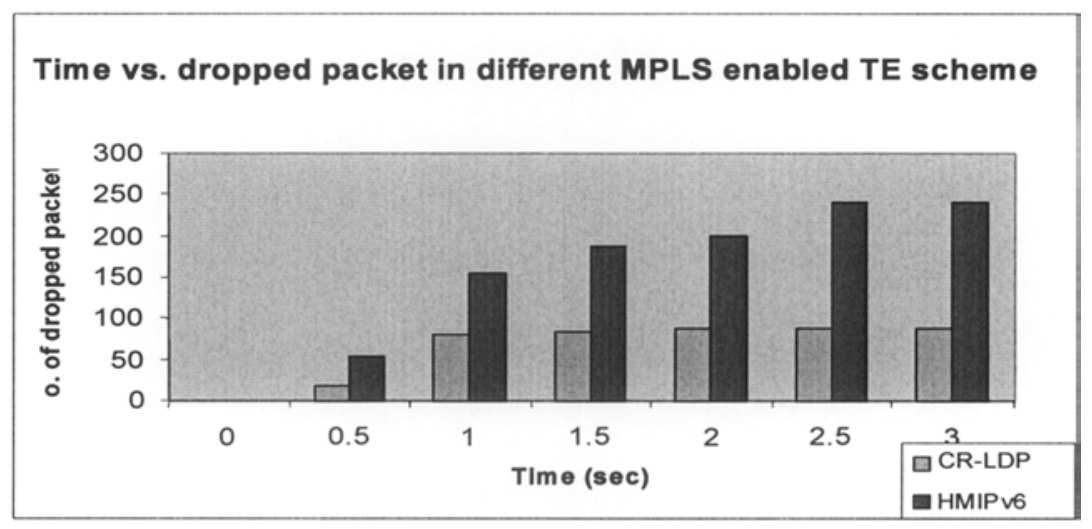

Figure 7. Packet Loss scenario

\section{Conclusion}

In this paper, we propose and evaluate an integrated architecture based on MPLS 3GWLAN-integrated network. With the demand for multimedia services in wireless environment, faster packet forwarding is required along with enhanced throughput and reduced packet loss. MPLS integration with HMIPv6 for the proposed integration model satisfies the all criterion that is validated through the extensive simulation work. Use of MPLS with traffic engineering like CR-LDP may ensure QoS within the 
domain. Thus with increasing mobile users MPLS based integrated network model would provide seamless connectivity with less packet loss, less congestion due to faster switching and increased throughput compared to only HMIPv6 based model. Established HMIPv6 protocol reduces network signaling overhead and handoff delay. Use of MPLS further enhances the performance as clear from the simulated results.

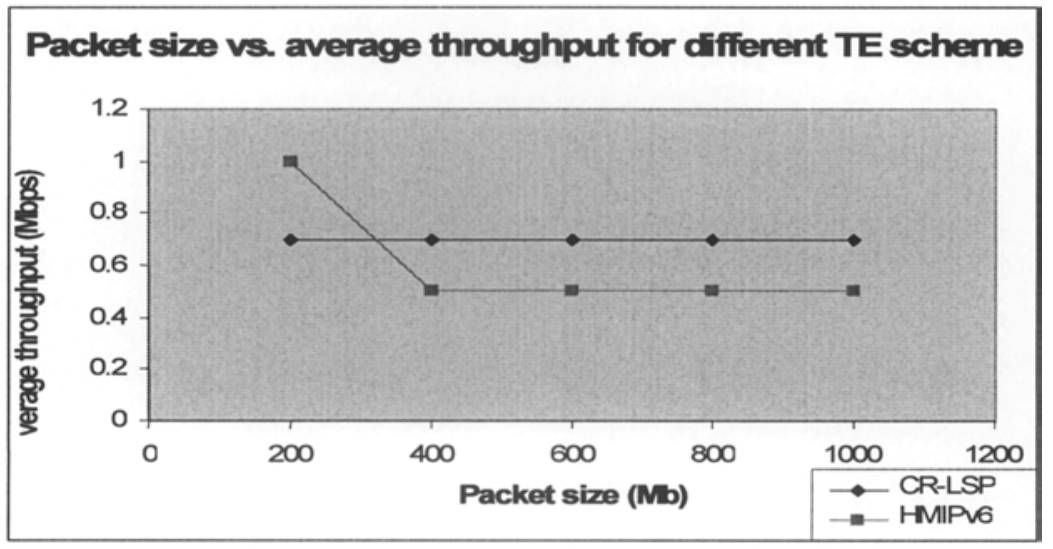

Figure 8. Throughput with increased packet size

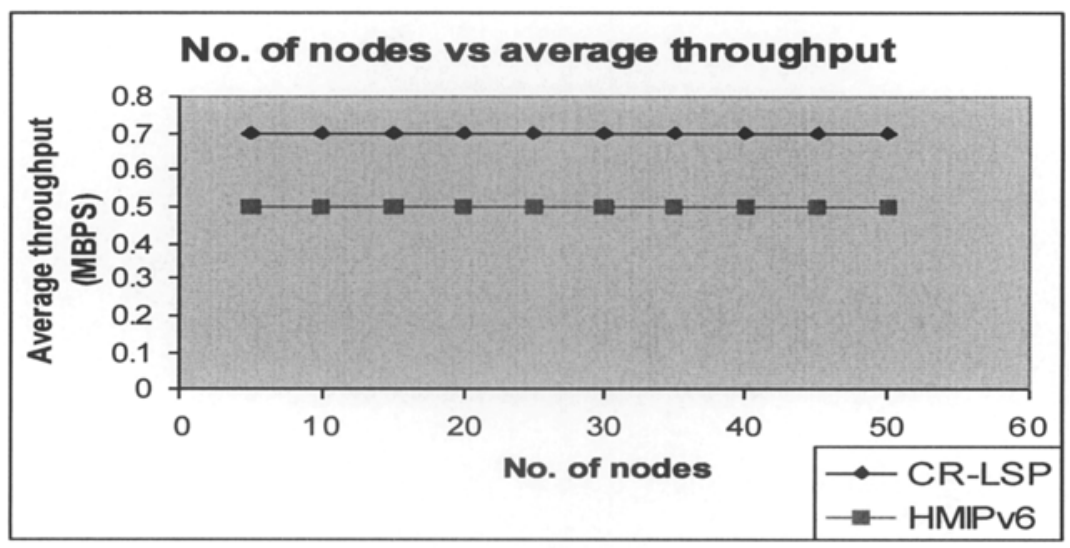

Figure 9. Throughput vs. number of nodes

Acknowledgement: Author Iti Saha Misra is thankful to AICTE for the financial support of this research work under AICTE, CAYT scheme, India. 


\section{References:}

1. T.S Rappaport, "Wireless Communications- Principles and Practice"- Prentice Hall of India Private Ltd.

2. Jeffrey Bannister, Paul Mather and Stebastian Coope, "Convergence Technologies for 3G Networks - IP, UMTS, EGPRS and ATM", John Wiley and Sons, Ltd.

3. Muhammed Jassemuddin," Architecture for Integrating UMTS \& 802.11 WLAN Network", IEEE Symposium on Computer Communications ISCC, Turkey, 2003, pp.1-8.

4. Milind M. Buddhikot, Girish Chandranmenon, Seungjae Han, Yui-Wah Lee, Scott.Miller, Luca Salgarellie, " Design and Implementation of a WLAN/CDMA 2000 Internetworking Architecture", IEEE communication Magazine, November 2003, pp.93-97.

5. C. Perkins, Ed., " IP mobility support", IETF RFC 2002, Oct, 1996.

6. Campbell et. al, "Design, implementation and evaluation of cellular IP", IEEE Personal Communication, Aug . 2002

7. R. Prasad et. al " IP Based Access Network Infrastructure for Next Generation Wireless Data Networks" IEEE Personal Communications, Aug. 2000.

8. S. Das et., al, "Tele MIP : Tele communication enhanced mobile IP architecture for fast intra domain mobility", IEEE Personal Communication, Aug. 2000.

9. D.Johnson, C.Perkin \& J.Arkho, " Mobility support in IPv6".

10. S. Jha and M. Hassan, Engineering Internet QoS, Artech House, 2002.

11. Fabino M. Chiussi, Denis A.Khotimsky, Santosh Krishnan, Lucent Technologies, "Mobility Management in 3G All IP Networks", IEEE Communication Magazine, September 2002.

12. Kaiduan Xie, Victor C.M Leung, "A MPLS Framework for Macro and Micro Mobility Management". pp.549-555, Orlando,FL, USA,March2002.

13. Tingzhou Yang, Yixin Dong, Bin Zhou, Dimitrios Makrakis, "Profile Based Mobile MPLS Protocol", Proc. of IEEE Canadian Conference on Electrical and Computer Engineering, 2002.

14. Tingzhou Yang, Yixin Dong, Bin Zhou, Dimitrios Makrakis, "Mobility Support for Differentiated Services in Next Generation MPLS Based Wireless Networks",www.site.uottawa.ca/ tyang/paper/mobility_for_diffserv.pdf.

15. Jesus Hamilton Ortiz, "Integration of Protocols HMIPv6 and M-RSVP over MMPLS in order to Provide QoS in IP Network Mobility", University Polytechnic of Madrid, Spain, ETSI Telecommunication.

16. Iti Saha Misra, Sudipta Dey and Debashis Saha ," 4G All IP Integration Architecture for Next Generation Wireless Internet", ICWN'05.

17. Iti Saha Misra, Chandi Pani," MPLS/HMIPV6 based mobility management framework for GPRS/WLAN integration architecture," IEEE conference on Wireless and Optical Communication Network, April 2006.

18. White paper (Juniper Networks) "Multiprotocol Label Switching: Enhancing Routing in the new public Network"

19. Xipeng, Xiao, Alan Hannan, Brook Bailey, "Traffic Engineering with MPLS in the Internet”, IEEE Network Magazine, Mar. 2000 
20. Youngseok Lee, Yongho Seok, Yanghee Choi, Changhoon Kim, "A Constrained Multipath Traffic Engineering Scheme for MPLS Networks", Proc. of IEEE Int. Conf. ICC2002. New York.

21. Vasu Jolly, Shahram Latifi, "An Overview of MPLS and Constraint Based Routing",

Ee.unlv.edu/venkim/opnet/AN\%20OVERVIEW\%20OF\%20MPLS\%20AND\%20 CONSTRAINT\%20BASED\%20ROUTING.pdf.

22. Shyam Subramanian and Venkatesan Muthukumar, "Alternative Path Routing Algorithm for traffic Engineering",www.ee.edu/venkim/opnet/Alternative_Path_Routing.pdf.

23. J.C. Chen and T. Zhang, IP Based Next Generation Wireless Networks- Wiley Publication, 2004

24. ns-2 home page, http://isi.edu/nsman/ns. 